\title{
Personalized Management of Cardiovascular Disorders
}

\author{
Kewal K. Jain \\ Jain PharmaBiotech, Basel, Switzerland
}

\section{Significance of the Study}

- The current trend in medicine is improving the precision of molecular diagnostics and biomarkers to guide the treatment of diseases by targeted delivery of pharmaceuticals and biological therapies. The diversity of the responses of individual patients to the standard treatment protocols is being increasingly recognized. Application of principles of personalized medicine will facilitate the translation of advances in "omics," such as genomics and proteomics, along with biotechnologies for improving the safety and efficacy of various treatments and prevention of disease. This review provides examples of strategies and integration of technologies to personalize the management of common cardiovascular disorders such as ischemic heart disease and hypertension.

\section{Keywords}

Cardiovascular diseases · Coronary heart disease . Heart failure · Hypertension · Personalized cardiology · Personalized medicine

\begin{abstract}
Personalized management of cardiovascular disorders (CVD), also referred to as personalized or precision cardiology in accordance with general principles of personalized medicine, is selection of the best treatment for an individual patient. It involves the integration of various "omics" technologies such as genomics and proteomics as well as other new technologies such as nanobiotechnology. Molecular diagnostics and biomarkers are important for linking diagnosis with therapy and monitoring therapy. Because CVD involve perturbations of large complex biological networks, a systems biology approach to CVD risk stratification may be used for improving risk-estimating algorithms, and modeling of personalized benefit of treatment may be helpful for
\end{abstract}

\begin{tabular}{ll}
\hline KARGER & $\begin{array}{l}\text { ( } 2017 \text { The Author(s) } \\
\text { Published by S. Karger AG, Basel }\end{array}$ \\
$\begin{array}{l}\text { E-Mail karger@karger.com } \\
\text { www.karger.com/mpp }\end{array}$ & $\begin{array}{l}\text { This is an Open Access article licensed under the Creative Commons } \\
\text { Attribution-NonCommercial-4.0 International License (CC BY-NC) } \\
\text { (http://www.karger.com/Services/OpenAccessLicense), applicable to } \\
\text { the online version of the article only. Usage and distribution for com- } \\
\text { mercial purposes requires written permission. }\end{array}$
\end{tabular}

guiding the choice of intervention. Bioinformatics tools are helpful in analyzing and integrating large amounts of data from various sources. Personalized therapy is considered during drug development, including methods of targeted drug delivery and clinical trials. Individualized recommendations consider multiple factors - genetic as well as epigenetic - for patients' risk of heart disease. Examples of personalized treatment are those of chronic myocardial ischemia, heart failure, and hypertension. Similar approaches can be used for the management of atrial fibrillation and hypercholesterolemia, as well as the use of anticoagulants. Personalized management includes pharmacotherapy, surgery, lifestyle modifications, and combinations thereof. Further progress in understanding the pathomechanism of complex cardiovascular diseases and identification of causative factors at the individual patient level will provide opportunities for the development of personalized cardiology. Application of principles of personalized medicine will improve the care of the patients with CVD.

(C) 2017 The Author(s) Published by S. Karger AG, Basel
Prof. K.K. Jain, MD, FRACS, FFPM

CEO, Jain PharmaBiotech

Bläsiring 7

$\mathrm{CH}-4057$ Basel (Switzerland)

E-Mail jain@pharmabiotech.ch 


\section{Introduction}

Personalized medicine simply means the prescription of specific treatments and therapeutics best suited for an individual, taking into consideration both genetic and environmental factors that influence the response to therapy [1]. The term "precision medicine" is used because diagnostic, prognostic, and therapeutic strategies are precisely tailored to each patient's requirements [2]. However, it does not cover the full scope of personalized medicine. Genomic/proteomic technologies have facilitated the development of personalized medicines but other technologies such as metabolomics are also contributing to this effort. "Personalized medicine is the best way to integrate new biotechnologies into medicine for improving the understanding of pathomechanisms of diseases and management of patients" [1]. This approach applies to personalized cardiology as shown in Figure 1.

The constantly growing volume of available data on the pathophysiology of cardiovascular disorders (CVD) will require an organized interpretation of variations in DNA and mRNA as well as proteins, on both the individual and the population level. Advances in biotechnologies have been applied to improve the diagnosis and treatment of CVD [3]. Although medicine is an art, personalized approaches place it on a scientific foundation [4].

\section{Role of Diagnostics in Personalized Management of Cardiovascular Disease}

Cardiac imaging includes the application of CT, MRI, and PET, and coronary angiography and scintigraphy of the heart are used routinely in the diagnosis of CVD. Molecular diagnosis is important for diseases with a genetic component and for understanding the pathomechanisms of various diseases, particularly using of molecular imaging.

\section{CVD with a Genetic Component}

Several cardiovascular diseases are recognized to have a genetic component, and examples of genes associated with cardiovascular diseases are listed in Table 1. Molecular genetics has contributed to the development of molecular cardiology by revealing new pathways to the diagnosis, prevention, and treatment of some cardiovascular diseases. Genetic approaches have helped to define the molecular basis of an increasing number of heart diseases, such as hypertrophic cardiomyopathy and long-QT syndrome (Brugada syndrome), a potentially fatal cardiac disorder associated with serious arrhythmias. Mutations in 5 genes that encode cardiac potassium ion channels have been identified and support the hypothesis that long-QT syndrome results from delayed myocellular repolarization. The diagnosis of long-QT syndrome and other channelopathies by an ECG is often difficult and

Fig. 1. Integration of technologies for personalized cardiology. Several technologies are integrated for the development of personalized cardiology. These include "omics" such as genomics and proteomics, molecular diagnostics, and biological therapies such as cell and gene therapies. Both genetics and epigenetic factors contribute. Some of the concepts of personalized medicine are like those of systems biology. Copyright: Jain PharmaBiotech.

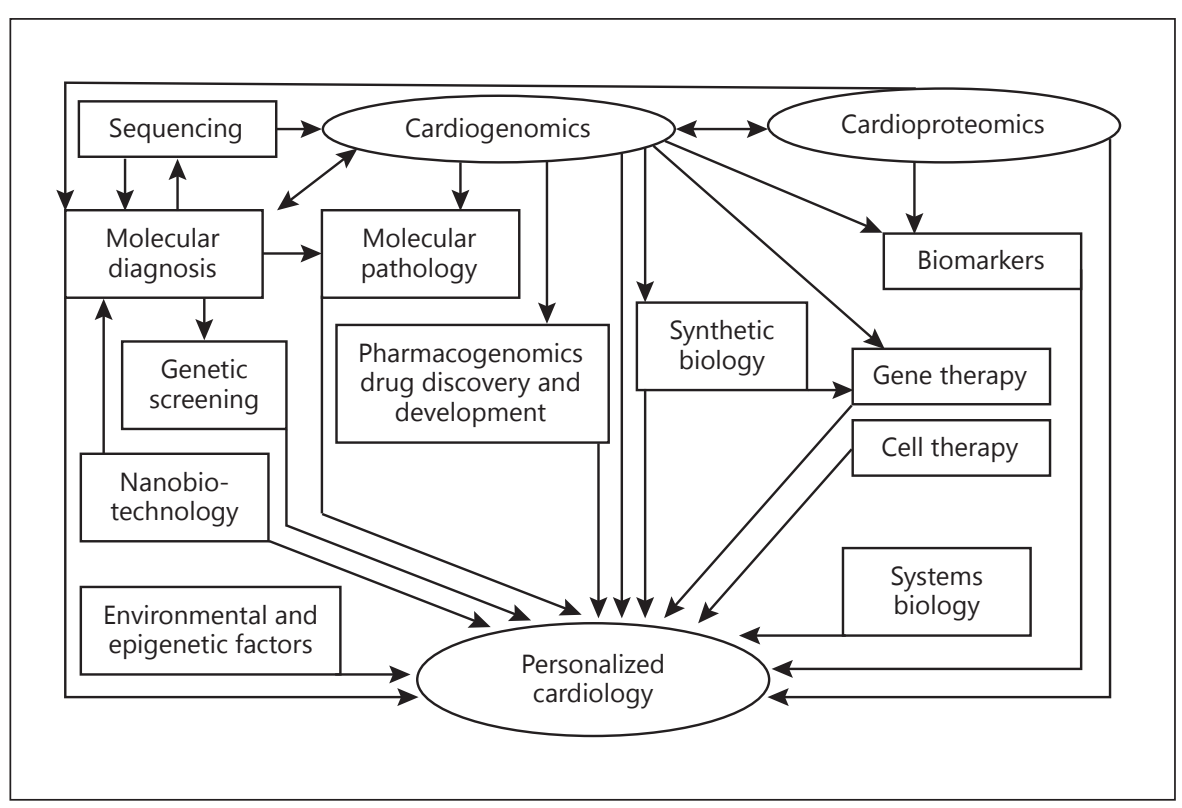


may be missed, which leaves the patient at risk for sudden cardiac death. FAMILION ${ }^{\mathrm{TM}}$, a comprehensive genetic test for heart rhythm disorders, examines 5 ion channel mutations to guide the physician in determining the best treatment options for those who are genetically predisposed to potentially fatal cardiac arrhythmias. The options include lifestyle modifications, prescription or avoidance of specific classes of drugs, or implantation of a defibrillator. The patient's family members also benefit from the test because it can identify if they have inherited the same mutation and may be at risk for a potentially fatal arrhythmia.
Gene Mutations Associated with the Risk of Coronary Heart Disease

Plasma triglyceride levels are heritable and correlated with the risk of coronary heart disease (CHD). Sequencing of the protein-coding regions of the human genome, i.e., the exome, in participants of European or African ancestry in the Exome Sequencing Project showed that rare mutations in the coding sequence, individually or in aggregate within a gene, were associated with plasma triglyceride levels [5]. Rare mutations in the gene encoding apolipoprotein C3 (APOC3) were associated with lower plasma triglyceride levels. The risk of $\mathrm{CHD}$ among carriers of any rare APOC 3 mutation was $40 \%$ lower than the

Table 1. Genes that cause cardiovascular diseases

\begin{tabular}{|c|c|c|c|}
\hline Category & Disease & Gene & Function \\
\hline $\begin{array}{l}\text { Congenital } \\
\text { malformations }\end{array}$ & $\begin{array}{l}\text { Atrial septal defect } \\
\text { Holt-Oram syndrome (holes } \\
\text { between the atria) }\end{array}$ & $\begin{array}{l}\text { NKX2-5 } \\
\text { TBX5 }\end{array}$ & $\begin{array}{l}\text { Transcription factor } \\
\text { Transcription factor }\end{array}$ \\
\hline \multirow[t]{2}{*}{ Cardiomyopathy } & $\begin{array}{l}\text { Familial hypertrophic } \\
\text { cardiomyopathy }\end{array}$ & $\begin{array}{l}\beta \text {-Myosin } \\
\text { Troponin T } \\
\text { Troponin I } \\
\text { Cardiac myosin-binding } \\
\text { protein C } \\
\text { a-Tropomyosin }\end{array}$ & $\begin{array}{l}\text { Muscle contraction } \\
\text { (forced generation) }\end{array}$ \\
\hline & $\begin{array}{l}\text { Idiopathic dilated } \\
\text { cardiomyopathy }\end{array}$ & $\begin{array}{l}\text { Actin } \\
\text { Dystrophin }\end{array}$ & $\begin{array}{l}\text { Muscle contraction } \\
\text { (force transduction) }\end{array}$ \\
\hline \multirow[t]{3}{*}{ Cardiac arrhythmias } & Long-QT syndrome & $\begin{array}{l}\text { KLVQT1 } \\
\text { HERG } \\
\operatorname{minK}\end{array}$ & Potassium channel \\
\hline & $\begin{array}{l}\text { Idiopathic ventricular fibrillation } \\
\text { (Brugada syndrome) }\end{array}$ & SCN5A & Sodium channel \\
\hline & $\begin{array}{l}\text { QT-related cardiac arrhythmia } \\
\text { with sudden death }\end{array}$ & NOS1AP & $\begin{array}{l}\text { The gene is the regulator of neuronal nitric } \\
\text { oxide synthase, which modulates cardiac } \\
\text { repolarization }\end{array}$ \\
\hline \multirow[t]{2}{*}{ Myocardial infarction } & Early onset & VAMP8 & Platelet degranulation \\
\hline & Early onset & HNRPUL1 & Encodes a ribonuclear protein \\
\hline Heart failure & Congestive heart failure & KIF6 wild-type gene & Kinesin family member 6 \\
\hline Hypertension & Essential hypertension & AGT & Contraction of arterial smooth muscle \\
\hline \multirow[t]{2}{*}{ Blood lipid disorders } & Familial hypercholesterolemia & LDL & Regulation of low-density lipoprotein \\
\hline & Familial dyslipoproteinemias & ApoE & Regulation of plasma lipid concentrations \\
\hline \multirow[t]{2}{*}{ Atherosclerosis } & Coronary artery disease & E-S128R & $\begin{array}{l}\text { Monitors white blood cell adhesion to the } \\
\text { arterial wall }\end{array}$ \\
\hline & $\begin{array}{l}\text { Coronary artery inflammatory } \\
\text { disease }\end{array}$ & $\begin{array}{l}\text { Interleukin-1 receptor } \\
\text { antagonist (IL-1ra) gene }\end{array}$ & $\begin{array}{l}\text { IL-1ra is a potent natural mechanism for } \\
\text { controlling IL-1 and inflammation }\end{array}$ \\
\hline Thrombotic disorders & $\begin{array}{l}\text { Venous thrombosis } \\
\text { Stroke }\end{array}$ & $\begin{array}{l}\text { Factor V } \\
\text { (Leiden mutation) }\end{array}$ & $\begin{array}{l}\text { Procoagulant normally by activated } \\
\text { protein C }\end{array}$ \\
\hline
\end{tabular}


risk among noncarriers. Another sequencing study from Denmark found that 3 rare variants of APOC3 - i.e., $\mathrm{R} 19 \mathrm{X}, \mathrm{IVS} 2+1 \mathrm{G} \rightarrow \mathrm{A}$, and A43T - are associated with substantially reduced levels of nonfasting triglycerides and a reduced risk of ischemic cardiovascular disease in the general population [6]. One limitation of this study is that, although the risk of ischemic cardiovascular disease is consistently inversely related to plasma levels of HDL cholesterol in observational studies, clinical trials as well as genetic studies involving Mendelian randomization have failed to establish a causal link between plasma levels of HDL cholesterol and the risk of ischemic CVD. Nevertheless, the findings of this study are of potential clinical importance because they suggest that APOC3 is a relevant drug target for reducing the residual cardiovascular risk, which was further supported by the reduction of plasma levels of APOC3 as well as triglycerides in animal models and in a phase I human clinical trial by inhibition of APOC3 by antisense oligonucleotides [7].

Plasma levels of non-HDL cholesterol are causally linked to the risk of CHD and they are a better predictor of cardiovascular risk than levels of LDL cholesterol alone because non-HDL cholesterol comprises all known cholesterol-containing lipoproteins that can cause atherosclerosis, including LDL, very-low-density lipoprotein, intermediate-density lipoprotein, chylomicrons and their remnants, and lipoprotein. Genetic evidence suggests a causal association between ASGR1 (asialoglycoprotein receptor), a gene encoding the major subunit of ASGR1 and non-HDL cholesterol. Sequencing of the genomes of Icelanders identified a rare 12-bp deletion (del12) in intron 4 of ASGR1, which was associated with an average reduction of $13.6 \mathrm{mg} / \mathrm{dL}$ in plasma levels of non-HDL cholesterol as compared with the average level in the general population [8]. ASGR1 is a link between the sialylation pathway, plasma levels of non-HDL cholesterol, and CHD. This association may suggest a new path to the development of future therapies for the prevention of CHD [9].

\section{Testing for Genetic Risk Factors as a Guide to Statin Therapy for $C H D$}

Statins are used as cardioprotective agents in persons with risk factors for CHD. An analysis of randomized controlled trials of both primary and secondary prevention using statin therapy led to a genetic risk score that identified individuals at an increased risk for both incident and recurrent $\mathrm{CHD}$ events [10]. Individuals with the highest burden of genetic risk derived the largest relative and absolute clinical benefits with statin therapy.
Carriers of the KIF6 (kinesin family member 6) wildtype gene are 50-55\% more likely to develop CHD. KIF6 as a biomarker of CHD is the basis of a genetic test, i.e., StatinCheck, which can be used to identify an increased risk for CHD and start treatment with statins. A study of 35 genetic polymorphisms associated with cardiovascular disease in the CARE (Cholesterol and Recurrent Events) trial and with CHD in the WOSCOPS (West of Scotland Coronary Prevention Study) showed that carriers of the KIF6 719Arg allele had an increased risk of coronary events, which was substantially reduced by pravastatin treatment [11]. Carriers of the 719Arg allele of KIF6 had a $34 \%$ higher risk of $\mathrm{MI}$ and a $24 \%$ higher risk of $\mathrm{CHD}$ compared to noncarriers among 25,283 women from the Women's Health Study, confirming and extending previous reports [12]. A CE-marked KIF6 genotyping assay is available to detect a genetic biomarker that may be used in conjunction with clinical evaluation and patient assessment to identify individuals at risk for $\mathrm{CHD}$ and to treat patients with elevated cholesterol for whom statin treatment is being considered.

\section{Next-Generation Sequencing for the Management of CVD}

PCR-based strategies are usually inadequate for genomic investigations involving many candidate genes. Next-generation sequencing (NGS) has overcome such limitations so that comprehensive testing is now feasible for clinically complex cases. Whole-genome sequencing is currently used only for highly selected, clinically complex cases but, with rapidly dropping test costs and increasing accuracy, it will eventually replace most, if not all, currently offered genetic tests [13]. An NGS procedure associated with DNA sequence capture has been reported that can sequence 202 cardiomyopathy-related genes simultaneously and a complementary data analysis pipeline can select and prioritize genetic variants [14]. By using this procedure, hypertrophic cardiomyopathy patients can be analyzed in a shorter time and at a lower cost than with current procedures. The specificity of the NGSbased procedure is at least as good as that of other techniques routinely used for mutation searching, and its sensitivity is much better. It will facilitate a personalized approach to treatment.

\section{SNP Genotyping in CVD}

Typing of specific SNP in the genome of an individual helps to diagnose or detect susceptibility to cardiovascular disease. Over 2,000 genes are linked to disorders involving the cardiovascular system including hyperten-
402
Med Princ Pract 2017;26:399-414 DOI: $10.1159 / 000481403$
Jain 
sion (HPN), myocardial infarction (MI), heart failure (HF), stroke, insulin resistance, metabolic disorders, dyslipidemia, and inflammation. A genotype score of 9 validated SNP that are associated with modulation in levels of LDL or HDL cholesterol is an independent risk factor for cardiovascular disease [15]. The score does not improve risk discrimination but modestly improves clinical risk reclassification for individual subjects beyond standard clinical factors.

\section{Testing Patients with Ischemic and Nonischemic}

\section{Heart Disease}

Blood flow (and thus oxygen) is often restricted to the heart muscle in ischemic heart disease because the heart cannot pump normally due to narrowing of the coronary arteries. In nonischemic forms of the disease, the heart cannot pump normally because the heart muscle has often become enlarged for other reasons such as physical deformity or alcohol abuse. Both conditions can lead to cardiac arrest or more gradual HF as the muscle weakens over time. Differentiation between the two types is important for planning the management. The next step is to develop a test that can be used in a clinical setting. Ischemic patients need to be monitored more closely in case they no longer respond to drugs and require surgery to unblock clogged arteries. Knowing which patients to treat and how closely to monitor them would be helpful for the physicians and improve health outcomes.

Lp-PLA2 (lipoprotein-associated phospholipase A2) is an enzyme that is implicated in the vascular inflammatory pathway that leads to plaque formation and atherosclerosis. Elevated Lp-PLA2 and C-reactive protein (CRP), biomarkers of inflammation, may be additive in their ability to predict the risk of CHD.

Routine cholesterol tests account for only about half of the predictability of heart disease. Determination of an expanded cholesterol profile provides detailed measurements of cholesterol and lipids, which play important roles in the development of cardiovascular disease. Such a profile can identify twice the number of people at risk for heart disease than traditional cholesterol tests and provide physicians with a basis for developing individualized treatment plans while continuing to track patients' progress in battling heart disease.

\section{Companion Diagnostics for Therapy of CVD}

Dalcetrapib, a cholesteryl ester transfer protein inhibitor, boosted levels of HDL cholesterol but did not affect the risk of cardiovascular events in a large morbidity and

Personalized Cardiology mortality trial. To determine if it may still have clinical benefits, a genetic analysis was done in the dal-OUTCOMES trial as well as the dal-PLAQUE-2 imaging study and it showed that individuals homozygous for the variant in the ADCY9 gene responded to dalcetrapib treatment with a significant reduction in cardiovascular risk [16]. A companion diagnostic is being used to identify patients with this genetic profile in a phase III trial of dalcetrapib.

\section{Biomarkers and Personalized Management of CVD}

The cardiovascular therapeutic area is complex and includes several overlapping diseases. In the past, low-cost biomarkers, such as blood pressure (BP) and cholesterol measurements were used. Clinical biomarkers for cardiovascular diseases have been revealed by intravascular ultrasound and in vivo tests for plaque composition and stability using imaging. Many new biomarkers have been discovered in recent years as a basis for diagnostic tests as well as for drug discovery and development [17]. There is a need for better diagnostic tests including those encompassing metabolic syndrome - a constellation of disorders including cardiovascular diseases, diabetes, and obesity. Biomarkers will be important for the development of personalized therapies for CVD.

\section{Pharmacogenomics/Pharmacogenetics of CVD}

Application of pharmacogenomics/pharmacogenetics for the development of personalized treatment for CVD is illustrated by examples of MI, HF, and HPN. The factors that should be considered when selecting the drug therapy for a patient with CVD include age, race, concomitant diseases, medications, and renal as well as hepatic functions. The renin-angiotensin system plays a major role in the development and progression of CVD by promoting vasoconstriction, sodium reabsorption, cardiac remodeling, norepinephrine release, and other potentially detrimental effects. Angiotensin-converting enzyme (ACE) inhibitors and angiotensin II type 1 receptor (AT1R) blockers are recommended for managing CVD such as HPN, myocardial ischemia, and HF. However, there is substantial variability in individual responses to these agents. An effort should be made to identify genes and gene products involved in differential responses to cardiovascular drugs as well as environmental influences on them.

Med Princ Pract 2017;26:399-414 


\section{Nanobiotechnology-Based Personalized Therapy of Cardiovascular Diseases}

Nanobiotechnology-based systems play an important role in the development of personalized medicine as they enable diagnosis of the pathology as well as treatment with a targeted delivery of therapeutics $[18,19]$. Biomarkertargeted nanoparticles can be used for molecular imaging as well as for pharmacologic modification of vulnerable atherosclerotic lesions that cause MI [20]. The potential dual use of nanoparticles for both imaging and site-targeted delivery of therapeutic agents to the cardiovascular disease offers great promise for individualizing therapeutics. Image-based therapeutics with site-selective agents should enable verification that the drug is reaching the intended target and a molecular effect is occurring.

Targeted imaging of vascular inflammation or thrombosis with magnetic nanoparticles (MNP) as a contrast agent for MRI may enable an improved risk assessment of atherosclerosis by detecting plaques at high risk for acute complications [21]. Cell death in the heart can be imaged in vivo using annexin-labeled MNP, particularly AnxCLIO-Cy5.5 [22]. Experimental studies have shown the feasibility of a combination of diagnosis and therapy using MNP. In a study on mice, MNP conjugated with plasmid DNA expressing enhanced green fluorescent protein and coated with chitosan were injected into the tail vein and directed to the heart by means of an external magnet without the need to functionalize the nanoparticles, and their location was confirmed by fluorescent imaging [23]. Perfluorocarbon nanoparticles provide an opportunity for combining molecular imaging and local drug delivery in CVD. Ligands such as monoclonal antibodies and peptides can be cross-linked to the outer surface of perfluorocarbons to enable active targeting of biomarkers expressed within the vasculature.

The combination of targeted drug delivery and molecular imaging with MRI has the potential to enable serial characterization of the molecular epitope expression based on imaging readouts. Monitoring and confirmation of the therapeutic efficacy of the therapeutic agents at the targeted site would facilitate personalized medical regimens.

\section{Personalized Cell Therapy of CVD}

The availability of technologies to derive induced pluripotent stem cells from adult terminally differentiated human somatic cells has potential for personalized cell- based therapy of CVD that retains patient-specific "omics" data. These cells can be enabled to mature into a state more closely resembling the adult human heart and subtype specification of cardiomyocytes, i.e., into pacemaker, atrial, or ventricular cells [24]. This will also enable more precise modeling of diseases that preferentially affect either the ventricles or atria in addition to opening new prospects for creating patient-specific bioengineered pacemakers.

\section{Personalized Management of Chronic Myocardial Ischemia}

Chronic myocardial ischemia leads to a dramatic reduction in myocardial contractility and impaired activity of the ion pumps involved in myocardial contraction-relaxation processes.

There is still some controversy surrounding the selection of medical versus surgical therapy for long-term management of patients with stable chronic myocardial ischemia. Patients with coronary artery disease who have significant lesions or symptoms despite optimum medical therapy require mechanical revascularization with coronary artery bypass grafting (CABG), percutaneous coronary intervention (PCI), or both. CABG has been the predominant mode of revascularization for more than half a century and it is the preferred strategy for patients with multivessel disease, especially those with diabetes mellitus, left ventricular systolic dysfunction, or complex lesions. Significant technical advances in PCI have made it the preferred method of revascularization in patients with single-vessel or low-risk multivessel disease. Improvements in both CABG (including total arterial revascularization, off-pump CABG, and "no-touch" graft harvesting) and PCI (including newer-generation stents, adjunctive pharmacotherapy, and intracoronary imaging) mean that there will a need for some guidelines for selection of the procedure best suited for an individual patient. A "heart team" approach is strongly recommended to select an evidence-based, yet individualized, revascularization strategy for all patients with complex coronary artery disease [25]. Finally, regardless of the method of revascularization, an adjunctive medical therapy is important for all patients with coronary artery disease, and this should also be personalized.

\section{Management of Chronic Angina Pectoris}

Pharmacological management of chronic stable angina pectoris includes short- or long-acting nitrates, $\mathrm{Ca}$ 
channel blockers, and $\beta$-blockers. However, even their intensive use is not highly effective in preventing angina.

Ranolazine is a potent inhibitor of the late $\mathrm{Na}^{+}$current and improves oxygen consumption, diastolic dysfunction, and coronary blood flow. Its mechanism of action is markedly different from that of other antianginal drugs. By altering the intracellular $\mathrm{Na}$ level, ranolazine affects the $\mathrm{Na}$-dependent $\mathrm{Ca}$ channels and indirectly prevents the Ca overload that causes cardiac ischemia. Several randomized, double-blind, placebo-controlled trials have provided evidence that supports the approval of a sustained-release formulation of ranolazine for clinical use in chronic ischemic heart disease [26]. Compared to other antianginal drugs, ranolazine provides an anti-ischemic effect without hemodynamic changes such as bradycardia or hypotension. This enables safe use of ranolazine in addition to other drug classes, improving control of anginal symptoms and representing a useful option in the presence of several comorbidities such as diabetes. It may play a useful role in the personalized management of ischemic heart disease. Treatment with ranolazine has been shown to be generally well tolerated, although it remains contraindicated in severe renal failure or moderate to severe hepatic impairment. It also has potential drug interactions through CYP450 enzymes.

\section{Management of $\mathrm{HF}$}

\section{$\beta$-Blockers}

$\beta$-Blockers are recommended in addition to ACE inhibitors for the management of HF. A response to $\beta$ blocker therapy in HF has been associated with the ACE genotype. Increased angiotensin II concentrations associated with the D allele may cause increased activation of the sympathetic nervous system and patients with the $\mathrm{D}$ allele may derive significant benefits from pharmacologic interventions to decrease the sympathetic nervous system activity (e.g., $\beta$-blocker therapy).

Despite the proven efficacy of $\beta$-blockers, there are many reasons why so many patients with HF are not treated with these medications. One important reason is concern for adverse reactions, which occur in $25-43 \%$ of patients. Discontinuation of therapy is frequent due to hypotension, bradycardia, and worsening of HF. This has led to the study of genetic variants that determine the response to $\beta$-blockers. Polymorphisms in the gene coding for the CYP2D6 isoenzyme, which catalyzes the metabolism of $\beta$-blockers such as metoprolol, carvedilol, timolol, and propranolol, may also affect the $\beta$-blocker response.
It is possible that the CYP2D6-related genotype interacts with drug target polymorphisms (e.g., $\beta$-receptor polymorphisms) and polymorphisms in genes involved in pathophysiology (e.g., the ACE I/D polymorphism) to influence the overall response to $\beta$-blockers.

\section{Bucindolol}

Bucindolol is a $\beta$-blocker/sympatholytic agent used for the treatment of advanced HF. Its efficacy is modulated by polymorphisms in the primary target $\beta 1$ adrenergic receptor ( $\beta 1 \mathrm{AR}) 389$ Gly on cardiac myocytes and a secondary target modifier $\alpha 2 \mathrm{C}$ AR on cardiac adrenergic neurons. The former is associated with efficacy enhancement and the latter with loss of effect, creating the possibility of genotype combination interactions that can be measured by clinical trials. Thus, genetic factors underlying $\mathrm{HF}$ and the response to bucindolol can help to identify those patients who will benefit most from bucindolol treatment. A clinical trial identified subpopulations with enhanced ( $\beta 1 \mathrm{AR} 389$ homozygotes), intermediate ( $\beta 1389$ Gly carriers $+\alpha 2 \mathrm{C} 322-325 \mathrm{Wt}$ homozygotes), and no ( $\beta 1389$ Gly carriers $+\alpha 2 C 322-325$ Del carriers) efficacy [27]. If prescribed genetically, bucindolol can be the state of the art in HF treatment for most patients.

\section{BiDil}

Enalapril therapy led to a significant reduction in the risk of hospitalization for HF among white patients with left ventricular dysfunction, but not among black patients. This finding led to additional research on the efficacy of therapies for HF in black patients. The relatively high level of HF in the black population has been attributed, in part, to a lack of nitric oxide (NO). BiDil, a combination of isosorbide dinitrate and hydralazine, reduces the mortality in this population by restoring depleted NO levels and protecting NO that is formed naturally in vascular endothelial cells. Hydralazine is an antioxidant and vasodilator. Neither drug is indicated separately for HF. A clinical trial showed that the addition of a fixed dose of isosorbide dinitrate plus hydralazine to standard therapy for HF including neurohormonal blockers was efficacious and increased survival among black patients with advanced HF. The study was terminated early owing to a significantly higher mortality rate in the placebo group than in the group treated with the drug combination. BiDil was approved by the FDA in 2005 and became the first drug to be developed and marketed on the basis of demonstrated efficacy in black subjects. This paved the way for individualized medicines.

Med Princ Pract 2017;26:399-414 DOI: $10.1159 / 000481403$ 
The African American Heart Failure Trial (A-HeFT) and the FDA approval of BiDil for race-specific prescription stirred the debate about the scientific and medical status of race. An analysis was published of the factors influencing physicians' prescription of BiDil and whether exposure to the controversy has an impact on their therapeutic judgments about the drug [28]. Overall, physicians prescribe and are willing to prescribe BiDil more to black patients than to white patients. However, physicians' lack of awareness about the controversial scientific status of A-HeFT suggests the need for more efficient ways to convey scientific information about BiDil to clinicians. Furthermore, the uncertainties about determination of the clinical utility of BiDil for the individual patient raise questions about whether this specific race-based therapy is in patients' best interest.

\section{Management of HPN}

HPN is defined as a systolic BP of at least $140 \mathrm{~mm} \mathrm{Hg}$ and a diastolic BP of at least $90 \mathrm{~mm} \mathrm{Hg}$. Before starting therapy of HPN, it is important to identify treatable causes of HPN. Aldosterone-producing adenomas (APA) are found in $~ 5 \%$ of individuals with HPN. Gain-of-function mutations in KCNJ5 and apparent loss-of-function mutations in ATP1A1 and ATP2A3 have been reported to occur in APA [29]. The authors found that KCNJ5 mutations are common in APA resembling cortisol-secreting cells of the adrenal zona fasciculate, but they are absent in a subset of APA resembling the aldosterone-secreting cells of the adrenal zona glomerulosa. They performed exome sequencing of 10 zona glomerulosa-like APA and identified 9 with somatic mutations in either ATP1A1, encoding the $\mathrm{Na}^{+} / \mathrm{K}^{+}$ATPase al subunit, or CACNA1D, encoding Cav1.3. The ATP1A1 mutations all caused inward leak currents under physiological conditions, and the CACNA1D mutations induced a shift of voltage-dependent gating to more negative voltages, suppressed inactivation, or increased currents. Many APA with these mutations were $<1 \mathrm{~cm}$ in diameter and had been overlooked on conventional adrenal imaging. Recognition of the distinct genotype and phenotype for this subset of APA could facilitate diagnosis. In the past, it was difficult to detect some of the nodules on traditional CT scans because they are so small. However, now they can be easily detected and removed by keyhole surgery, completely curing HPN.

There is a great variation in the management of HPN. The level of BP at which HPN should be treated has been controversial. In 2014, evidence-based guidelines recom- mended that adults aged 60 years or older only need to take medication if their BP readings exceed 150/90 [30]. In 2015, results of the SPRINT trial suggested that targeting a systolic BP $<120 \mathrm{~mm} \mathrm{Hg}$ as compared to $<140 \mathrm{~mm}$ $\mathrm{Hg}$ in patients with a high risk of cardiovascular events but without diabetes resulted in lower rates of fatal and nonfatal major cardiovascular events and death from any cause, although significantly higher rates of some adverse events were observed in the intensive-treatment group [31]. Ideally, individual risks must be assessed in order to make the best decision regarding the treatment of a patient with HPN. Assessment identifies important cardiovascular risk factors that may warrant treatment and helps to enhance the benefits that patients can expect from personalized approaches. The benefits of treating hypertensive patients also vary depending on each patient's competing risks of dying from causes other than cardiovascular ones. For example, patients with multiple serious conditions, such as end-stage Alzheimer's disease, obstructive lung disease, frequent falls, gout, and urinary incontinence, have high competing risks that may minimize or negate the benefits of treating their HPN.

If the treatment of HPN is strictly based on BP levels, some patients receive too many medications and others too little. Individualized recommendations should consider multiple factors for patients' risk of heart disease, e.g., age, gender, smoking, etc. Use of medications should be guided by the patient's risk of these diseases and how much adding a new medication decreases that risk - not solely on their BP level. Those who have mild HPN but a high cardiovascular risk receive a lot of benefit from treatment, but those with a low overall cardiovascular risk do not. A study found that benefit-based tailored treatment was more effective and required less antihypertensive medication than current guidelines based on treatment to achieve specific BP goals [32]. Biomarkers may help to identify patients in whom HPN is linked to the risk of $\mathrm{CHD}$. For example, a high serum parathyroid hormone level is related to a high diastolic BP and it is also a risk factor for CHD [33]. Once the decision to treat HPN has been made, an appropriate therapy should be selected. HPN is a good model for the development of personalized medicine because it is a multifactorial disease.

\section{Adjusting Therapy of HPN to Fluctuations in BP}

$\mathrm{BP}$ is a continuous, not a static, variable. Individuals exhibiting similar clinical or home BP can differ considerably with respect to their average day- and nighttime val-
406

Med Princ Pract 2017;26:399-414 DOI: $10.1159 / 000481403$
Jain 
ues, sleep, and responses to mental and physical stimuli, as well as seasonal variations. Several such episodes of BP fluctuations increase the cardiovascular risk independently of the average of conventionally recorded BP readings. Antihypertensive drugs differ in their effects on intersession BP variability and the associated risk of stroke. Optimization of personalized cardiovascular risk assessment and attempts to reduce such risk involve the identification of BP variability that best estimates the individual cardiovascular risk [34]. There is a need to establishing "normal" and "high-risk" BP variability distributions to test the hypothesis that attenuating such variability via drug or device therapy reduces the cardiovascular risk more than $\mathrm{BP}$ reduction per se. The results of these studies should be integrated into clinical practice.

Another implication of BP fluctuations is that the dose and delivery of antihypertensive drugs should be adjusted to fluctuations of BP so that short-acting doses are delivered at the time of peak BP that is above the value according to the guidelines. It is preferable to maintaining $24-\mathrm{h}$ delivery of a drug with a prolonged release preparation. Adjusted delivery is feasible with a BP sensor linked to a transdermal patch with controlled release. Although transdermal drug delivery technology has advanced enough to accomplish this, BP sensor technology needs further development to make a system that is easy to use by the patient.

\section{Choice of Drugs for HPN}

Over 100 medications are available for the treatment of HPN in several categories: diuretics, $\alpha$-blockers, $\beta$ blockers, aldosterone antagonists, ACE inhibitors, angiotensin II receptor antagonists, CNS-active agents, and calcium channel blockers. Each of these categories contains several distinct drugs, which vary in their efficacy and liability to produce adverse reactions in different patient populations. $\beta$-Adrenergic antagonists are generally recommended as first-line therapy, along with thiazide diuretics, for the treatment of HPN. However, as many as $60 \%$ of hypertensive patients do not achieve adequate lowering of $\mathrm{BP}$ using monotherapy with $\beta$-blockers. It is plausible that genetic variation in the $\beta$-adrenergic receptor genes accounts for some of the observed variability in BP response.

Antihypertensive monotherapy does not address the multifactorial nature of $\mathrm{HPN}$ as a disease with many pathways. An approach to increasing the chances of efficacy is to use a fixed combination of drugs with different modes of action as the initial therapy in the treatment of HPN. The additive or synergistic effect of combination

Personalized Cardiology therapy may lower the $\mathrm{BP}$ in patients who tend to have less than a full response to one component only. This is still an approximate method and may increase the adverse effects of drug interactions unless the combination is selected individually for each patient.

\section{Control of BP with Vagal Nerve Stimulation}

Mechanoreceptors in the aortic arch relay BP levels through vagal nerve (VN) fibers to the brainstem and trigger the baroreflex, lowering the BP. Selective electrical stimulation of these nerve fibers reduces the BP in rats. A technique is needed to localize and stimulate these fibers inside the $\mathrm{VN}$ without inadvertent stimulation of nonbaroreceptive fibers causing side effects like slowing of the heart rate and breathing. Such a method for selective $\mathrm{VN}$ stimulation to reduce $\mathrm{BP}$ without the side effects has been demonstrated by localization of the baroreceptor compound activity of the rat $\mathrm{VN}$ using a multichannel cuff electrode, tripolar recording, and an averaging algorithm triggered by $\mathrm{BP}$ or an electrocardiogram [35]. In this study, tripolar stimulation of electrodes near the barofibers reduced BP without triggering a significant effect on heart rate and breathing. The BP drop was further adjusted to $60 \%$ of the initial value by varying the stimulation pulse width and duration, and it lasted up to 5 times longer than the stimulation. This method is independent of the electrode's relative position, does not damage the nerve, and can run on implantable, ultra-low-power signal processors. Its translation into the clinic will improve the control of BP without variations in individual responses to other methods and in keeping with personalized management of HPN.

\section{Correction of Causes and Risk Factors of HPN}

Several causes and risk factors of HPN have been identified and these should be corrected. A low-salt diet for salt-sensitive HPN and management of stress by relaxation and meditation are well known and should be incorporated into personalized lifestyle modification advice. A Mendelian randomization study showed that a low plasma 25-hydroxyvitamin D concentration is one cause of HPN and this is partially due to genetic variants associated with a low endogenous production of 25-hydroxyvitamin $\mathrm{D}$, raising the possibility of prevention or reduction of HPN with vitamin D supplementation [36]. This finding warrants further investigation in an independent, similarly powered study.

Med Princ Pract 2017;26:399-414

DOI: $10.1159 / 000481403$ 


\section{Genes and HPN}

There is an increasing interest in genes related to HPN. Genetic factors account for $40-50 \%$ of a person's susceptibility to HPN. HPN is more prevalent and contributes to more severe manifestations of cardiovascular disease in African Americans than in any other US ethnic group. Previous searches of the genome found limited evidence of genes that determine HPN. So far, most gene discovery studies have involved people of European descent. A landmark study involving nearly 30,000 African-Americans discovered 4 novel gene variations associated with $\mathrm{BP}$, which are also associated with $\mathrm{BP}$ across other populations [37]. Although how genes regulate $\mathrm{BP}$ is unknown, the findings contribute to a better understanding of $\mathrm{BP}$ pathways that can lead to the future development of a drug target for HPN and may guide therapy for clinical care. The authors of the study are conducting additional research to determine whether the 4 genes respond to the existing HPN medications.

A study of array-based profiles of the blood gene expression of individuals with known BP measurements identified transcripts with expression profiles that varied in concert with BP [38]. The identification of a "BP 6 gene signature" consisting of FOS, MYADM, PP1R15A, TAGAP, S100A10, and FGBP2 may represent a biomarker of susceptibility as well as a basis for the prevention and treatment of HPN.

As a polygenic disorder, HPN remains a challenge for designing better future treatments. Individuals typically respond differently to a given medication depending on which gene mutation they carry. The more information researchers gather, the greater the opportunity for clinicians to prescribe the drug that is most efficacious based on the patient's specific mutation.

Improving the Management of HPN by Targeting New Pathways

$\mathrm{BP}$ is maintained by electrical impulses from the brain that travel to the arteries via the sympathetic nervous system and control their diameter, but this pathway is often chronically overactive in HPN. Most antihypertensive drugs work by decreasing both acute and chronic activity in the sympathetic nervous system. However, these drugs often have serious side effects, such as fatigue, dizziness, and erectile dysfunction. These drawbacks have led to the search for novel ways to inhibit the sympathetic nervous system while causing fewer problems for patients with HPN.

A study found a new link between the brain and increased BP, a steroid called ouabain, and the pathway that connects the brain to ouabain's effects on proteins that regulate arterial calcium and contraction [39]. The proximal components of this axis are neuronal pathways activated by brain angiotensin II that depend on central aldosterone and mineralocorticoid receptors. The distal components of the axis include upregulated circulating levels of ouabain and related steroids, and functional reprogramming of arterial function due to increased expression of arterial myocyte proteins that raise arterial myocyte $\mathrm{Ca} 2+$ levels and myogenic tone, resulting in augmentation of sympathetic responses. These results are consistent with the idea that long-term increases in central angiotensin II and circulating ouabain sustain the BP via the combined effects of heightened sympathetic activity and functional reprogramming of arterial function. These findings suggest new approaches for treating HPN by modifying this pathway.

\section{Individualized Therapy of HPN Based on Risk Factors} of Heart Disease

Individualized recommendations for therapy of HPN consider multiple factors for patients' risk of heart disease. Patients who have mild HPN but a high cardiovascular risk profile receive considerable benefits from treatment, but those with a low overall cardiovascular risk do not. Individualized treatment of HPN could prevent $>25 \%$ of heart attacks and strokes while using less medications. Patients with HPN and hypercholesterolemia, who are treated for both concurrently, are $50 \%$ less likely to get heart disease [40].

Antihypertensive therapy has been clearly shown to reduce the risk of cardiovascular disease in those with vascular or renal disease, diabetes, or HPN with end-organ damage or, in the absence of these conditions, among persons with a systolic BP of $160 \mathrm{~mm} \mathrm{Hg}$ or higher. There are insufficient data to guide decisions about BP levels for the initiation of antihypertensive agents in persons at low or moderate cardiovascular risk who have mild uncomplicated HPN. The Heart Outcomes Prevention Evaluation (HOPE)-3 trial evaluated the role of therapy in persons at intermediate risk (defined as an annual risk of major cardiovascular events of $\sim 1 \%$ ) who do not have vascular disease and who have a systolic $\mathrm{BP}<160 \mathrm{~mm} \mathrm{Hg}$ [41]. The results showed that treatment of HPN was not associated with a lower rate of major cardiovascular events than placebo among persons at intermediate risk who did not have cardiovascular disease. Although no benefit of BP-lowering was observed overall, a prespecified subgroup analysis showed a $27 \%$ lower risk of cardiovascular events with BP-lowering therapy in the sub-
408

Med Princ Pract 2017;26:399-414 DOI: $10.1159 / 000481403$
Jain 
group of participants who were in the upper third of systolic BP levels (>143.5 $\mathrm{mm} \mathrm{Hg}$ ). Among the patients in that subgroup who received placebo, the rate of cardiovascular events was $6.5 \%$ over a period of 5.6 years [42]. These results may help to define the combined threshold of systolic BP $(<140 \mathrm{~mm} \mathrm{Hg})$ and cardiovascular risk $(<5.0 \%)$ below which the use of BP-lowering medications may not be useful in the short term. However, these results do not rule out the possibility of a benefit with longer-term treatment in a portion of this relatively low-risk population.

\section{Personalized Management of Hypertensive Patients} with Albuminuria

Hypertensive subjects with microalbuminuria and macroalbuminuria are more likely to have uncontrolled $\mathrm{BP}$ and a higher average SBP. Macroalbuminuria is associated with isolated uncontrolled SBP in non-Hispanic blacks and whites, and macroalbuminuria is associated with isolated uncontrolled SBP and DBP and a high average DBP only in non-Hispanic blacks [43]. Compared with non-Hispanic whites, non-Hispanic blacks and Mexicans have lower associations of microalbuminuria with uncontrolled BP and isolated uncontrolled SBP. Moreover, the association of microalbuminuria with uncontrolled BP is lower in non-Hispanic blacks and Mexicans than in non-Hispanic whites. These findings indicate that health providers need to improve care for mildly elevated albumin excretion rates in non-Hispanic white hypertensive patients while maintaining the quality of care in non-Hispanic blacks and Mexicans.

\section{Personalized Management of HPN in the Elderly}

Isolated systolic HPN is more common in the elderly than in younger adults and it is associated with poor outcomes such as cerebrovascular disease and acute coronary events. International guidelines are inconsistent in providing recommendations on optimal BP targets in hypertensive elderly patients because of the limited evidence in this population. Evidence from clinical trials supports the use of antihypertensive drugs in hypertensive elderly patients for reducing CVD and mortality. However, elderly participants in these trials may not be the typical elderly patients seen in clinical practice, and there are potential risks associated with the use of antihypertensive drugs in the elderly, such as cognitive decline, orthostatic hypotension, falls, and fractures [44]. Therefore, the benefits and risks of using antihypertensive medications need to be individually assessed in hypertensive elderly patients based on the available evidence.

Personalized Cardiology

\section{Personalized Management of HPN in Women}

A study contradicted the previous medical belief that the impact of HPN is the same in both sexes and concluded that a high BP may be significantly more dangerous in women than in men [45]. Knowledge of the hemodynamic factors that account for the development of HPN should help to tailor therapeutic approaches and improve BP control. Few data exist regarding sex differences in hemodynamic factors contributing to HPN progression among patients with untreated nondiabetic stage I and II prehypertension (PreHyp) as defined by the Joint National Committee-7 guidelines (JNC-7). In each of the $3 \mathrm{HPN}$ categories, the increased BP in men is associated with significant augmentations in stroke volume and cardiac output compared to women. Sexrelated hemodynamic differences are associated in women with higher plasma levels of leptin, hs-CRP, plasma angiotensin II, and serum aldosterone, and there are no differences in the serum concentrations of cytokinins. In women but not men, hs-CRP correlated with plasma concentrations of TGF- $\beta 1$ and body weight; in addition, plasma TGF- $\beta 1$ correlated with levels of serum vascular cell adhesion molecule 1 . This information is useful for selecting antihypertensive drugs as there are significant differences in the hemodynamic and hormonal mechanisms that account for the increased BP in women compared to men. Women should be treated for high BP earlier as well as more aggressively than men.

\section{Pharmacogenomics/Pharmacogenetics of Antihypertensive Drugs}

Genetic factors may influence the response to antihypertensive medication. Common genetic polymorphisms associated with the response to antihypertensive drugs such as diuretics, ACE inhibitors, angiotensin II antagonists, and $\beta$-blockers have been identified by genomewide studies, but there is no currently available pharmacogenomic test to guide HPN treatment in clinical practice [46].

Diuretics. These are often the first-line drugs for HPN but their overall efficacy is not sufficient. Many patients suffer adverse effects such as disturbances of serum $\mathrm{K}^{+}$ levels. Variations in efficacy and susceptibility to adverse reactions of diuretics may be partially caused by genetic polymorphisms of genes involved in the pharmacodynamics and pharmacokinetics of diuretics. Genes with a role in the pharmacokinetics of most diuretics are renal drug transporters, especially OAT1, OAT3, and OCT2 (genes SLC22A6, SLC22A8, and SLC22A2), whereas vari-

Med Princ Pract 2017;26:399-414 DOI: $10.1159 / 000481403$ 
ants in carbonic anhydrase, CYP450 enzymes, and sulfotransferases are relevant only for specific substances. Genes on the pharmacodynamic side include the primary targets of thiazide, loop, the $\mathrm{K}^{+}$-sparing and aldosteroneantagonistic diuretics NCC, NKCC2, and $\mathrm{ENaC}$, and the mineralocorticoid receptor (genes SLC12A3, SLC12A1, SCNN1A, B, G and NR3C2). Polymorphisms in these and in associated proteins, e.g., GNB3, $\alpha$-adducin, and ACE, seem to be clinically relevant.

Genetic alterations in hypertensive patients can dramatically increase the risk of a heart attack, stroke, or death and may explain why some hypertensive patients fare worse than others even if they take the same medication. Patients carrying the $\alpha$-adducin gene are less likely to suffer a heart attack or stroke if they are taking a diuretic. Data from the International Verapamil SR-Trandolapril Study (INVEST-GENES) suggested that one genotype group benefited from the diuretic and had a reduction in heart attacks and strokes, while the other genotype group did not [47]. The findings of this study may enable patients to receive appropriate personalized medicine based on their genetic makeup.

ACE Inhibitors. Polymorphism of the ACE gene is known to influence the response to the ACE inhibitor fosinopril in hypertensive patients. Blacks with HPN, as a group, have a lower plasma renin activity and are less likely than hypertensive whites to achieve adequate $\mathrm{BP}$ reductions with ACE inhibitor monotherapy.

Rostafuroxin. Two mechanisms, among others, are associated with essential HPN and related organ damage: mutant $\alpha$-adducin variants and high concentrations of endogenous ouabain. The antihypertensive agent rostafuroxin is a digitoxygenin derivative that selectively disrupts the mutant adducin and the ouabain-activated $\mathrm{Na}^{+} / \mathrm{K}^{+}$-pump binding to the Src-SH2 domain without affecting the binding of normal proteins. A clinical trial is ongoing to substantiate some of the following findings [48]: (i) one quarter of patients with primary HPN display mutations of adducin or concentrations of endogenous ouabain and would be expected to respond to therapy with rostafuroxin; and (ii) because the mechanisms that are inhibited by rostafuroxin also underlie HPN-related organ damage, this drug may also reduce the cardiovascular risk in these patients beyond that expected by the reduction of SBP alone. Confirmation of these findings will help in patient stratification for predicting the effectiveness of rostafuroxin (but not that of any other antihypertensive drug) in patients carrying key gene variants.

410

Med Princ Pract 2017;26:399-414 DOI: $10.1159 / 000481403$
Future of Pharmacogenomic/Pharmacogenetic-

Guided Therapy of HPN

Although there are controversies to settle and difficulties to overcome, pharmacogenomics/pharmacogenetics may yield successful strategies to optimize drug therapy. Several candidate genes are currently under investigation for their potential to modify the response to antihypertensive drugs. Findings from previous studies require conformation in other studies to be able to make definitive conclusions about current positive drug-gene interactions. It is also important that research groups collaborate more to facilitate the conduction of a meta-analysis for conclusive results. With the development of efficient methods for analyzing massive amounts of data, further studies may eventually lead to the optimization of antihypertensive drug therapy based on the genetic profiles of patients. Pharmacogenetics-guided therapy has clinical potential for the management of HPN, but there are few controlled studies on this topic.

The availability of such a practical pharmacogenomic test would enable the selection of the most effective drug as first-line treatment for HPN, leading to a reduction of the number of drugs required for adequate treatment as well the number of visits by the patient to the health care facility for BP monitoring. The overall effect would be improvement in the quality of health care and cost savings.

\section{Scheme for the Management of HPN via a Personalized Approach}

Despite the many therapeutic options for HPN, only $27 \%$ of the patients achieve adequate BP control. Therefore, there is an opportunity to improve the management of HPN via a personalized approach as shown in Figure 2.

\section{Clinical Trials of Personalized Therapy of Cardiovascular Diseases}

In the era of personalized medicine, biomarkers of cardiovascular diseases can be used to improve the efficiency of clinical trials by identifying the most appropriate patients to include. In the absence of a validated biomarker, a predictive biomarker response could be used to identify likely responders. In the section on personalized management of HF, examples were given of the use of subgroup analysis during follow-up of clinical trials of bucindolol and BiDil. Bucindolol is now being evaluated specifically in patients with the ADRB1 Arg389Arg genotype (Clini- 
Fig. 2. Scheme of a personalized approach to the management of hypertension. The scheme is a stepwise guide to the evaluation of a patient with hypertension. Both routine laboratory chemistry and molecular diagnosis such as genotyping are used. Findings guide the selection of management strategies, which can be changed depending on the response to treatment. Pharmacogenetics and pharmacokinetics are taken into consideration in selection of the drugs and the treatment is monitored. $\mathrm{BP}$, blood pressure; HPN, hypertension. Copyright: Jain PharmaBiotech

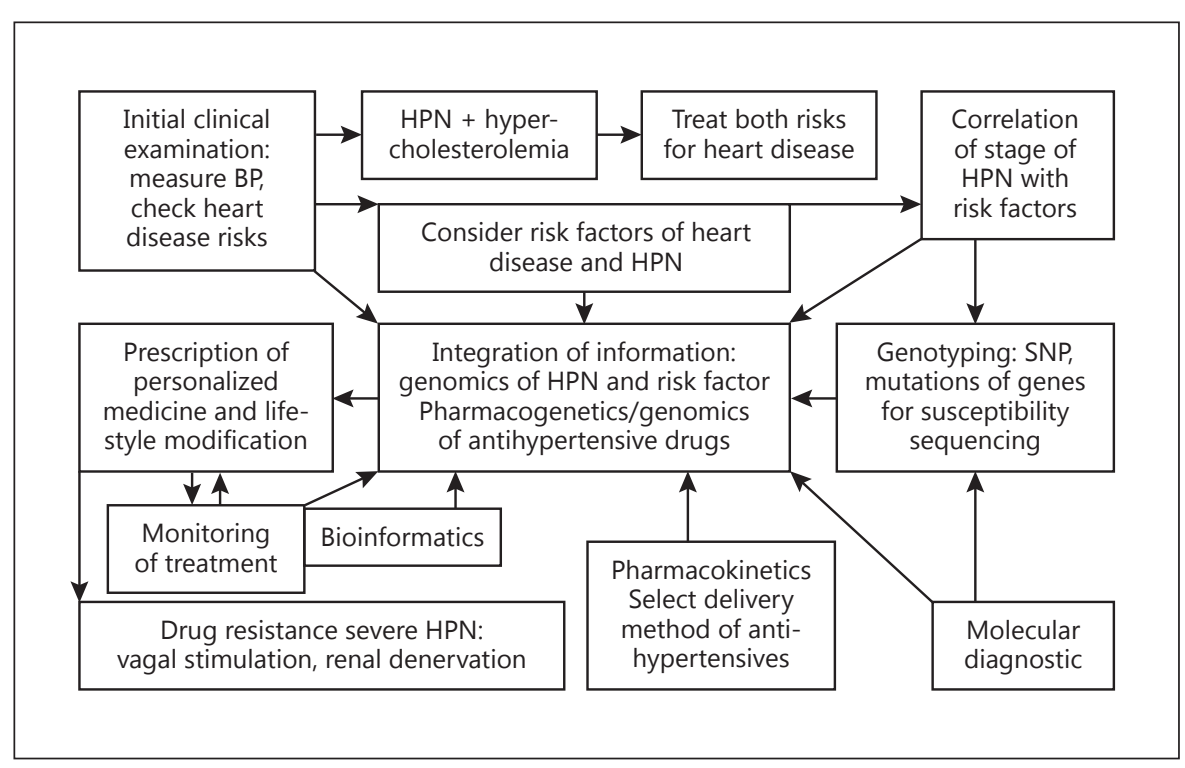

calTrials.gov identifier: NCT 01970501). In cases where exploratory data suggest that population subsets may respond differently, adaptive trial designs that use interim analyses may be useful to enrich the patient population. Modifications of the study population may also be made based on safety data from interim analyses.

\section{Modification of Lifestyle Factors in the Management of CVD}

Both genetic and lifestyle factors are key drivers of CHD. Genome-wide association analyses have identified $>50$ independent loci associated with the risk of CHD. These risk alleles, when aggregated into a polygenic risk score, are predictive of incident coronary events and provide a continuous and quantitative measure of genetic susceptibility. Quantification of both genetic and lifestyle risks in 3 prospective cohorts and 1 cross-sectional study revealed that adherence to a healthy lifestyle was associated with a substantially reduced risk of CHD within each category of genetic risk [49]. A healthy diet, exercise, smoking cessation, and stress reduction are recognized measures for reducing the risk of cardiovascular disease. A study compared the effectiveness of these lifestyle interventions for individual risk factors for reducing the 10year cardiovascular disease risk and found yoga and smoking cessation to be the most effective forms of cardiovascular disease prevention [50]. The benefit is enhanced when interventions are performed simultaneous-

Personalized Cardiology ly rather than as single measures. Personalized Activity Intelligence is an easily understandable and scientifically proven algorithm to inform users of how much physical activity is needed to reduce the risk of premature death from CVD [51].

\section{Systems Biology Approach to Personalized Cardiology}

A systems biology approach to CVD risk stratification may be employed to improve risk-estimating algorithms through the addition of high-throughput-derived "omics" biomarkers [52]. In addition, modeling of a personalized benefit-of-treatment may help to guide the choice of intervention. With the realization that CVD involves perturbations of large complex biological networks, future directions in drug development may involve moving away from a reductionist approach toward a system level approach. Accurate risk scores, more effective drugs and a personalized estimation of the benefit from treatment are 3 much needed tools for CVD prevention. A systems medicine approach can hopefully provide value in all of these areas. An interesting tool in systems medicine is protein-protein interaction (PPI) networks [53]. PPI networks have the potential to provide useful information in CVD, since each protein is placed in a larger network context and thus alterations in proteins in the diseased state can be compiled and translated into meaningful biological tasks. For example, if 100 different proteins are shown

Med Princ Pract 2017;26:399-414 411 
to be altered in the blood macrophages or endothelial cells of persons with CVD and 80 of them happen to be highly connected, shown by a PPI, then that part of the network and the related metabolic function could be concluded to be perturbed in the diseased state. Further on, if a few of the proteins are shown to interact with lots of the other disease-related proteins, these highly connected proteins might be central to the disease progression itself. Thus, PPI could identify central hubs in the disease network, i.e., hubs that might provide a pathophysiological understanding and be suitable as drug targets.

\section{Role of Bioinformatics in the Development of Personalized Cardiology}

Personalized medicine is often referred to as information-based medicine. Bioinformatics tools will help to integrate massive data from various technologies and sources of information for the development of personalized medicine and facilitate therapeutic decision-making by the physicians. The transition to electronic health records and the availability of patient data has led to increases in the volume and complexity of patient information that will require bioinformatic support for analysis. Computational diagnostics includes the identification of novel, molecularly defined entities of a disease. For many clinical decision problems, where numerous features are used to monitor a disease, neural networks and other machine-learning approaches can be helpful. Analysis of data gathered through digital technologies and wearable devices will facilitate the practice of personalized medicine. Wireless noninvasive biosensors can monitor vital signs including continuous BP and heart rhythm. A cell phone-sized device can be used to acquire high-resolution 2-D echocardiography and color flow and consumers can learn how to acquire their own echocardiograms and transmit the images to their physicians for real-time interpretation.

\section{Concluding Remarks and the Future of Personalized Cardiology}

Despite advances in molecular cardiology and diagnostics, there is limited translation of new technologies into clinical practice and few personalized therapies are available for CVD. The development of targeted therapies and personalized medicine approaches is difficult due to the complex etiology of common cardiovascular diseases.
Another problem is the classification of cardiovascular diseases, which is based on the clinical phenotype rather than on the underlying mechanism driving the disease. Further progress in the understanding of the pathomechanism of complex cardiovascular diseases and the identification of causative factors at the individual patient level will provide opportunities for the development of personalized medicine. The development of targeted therapies that directly mitigate the underlying cause of disease in patients in whom a single factor appears to be the driver, rather than treatment of the resulting clinical symptom, will advance personalized medicine for common cardiovascular diseases [54]. Individual responses to drugs vary and are partly determined by genes. Simple genetic analyses can improve response prediction and minimize the side effects of drugs such as warfarin and simvastatin. NGS will facilitate the identification of mutations causing cardiovascular diseases. In contrast to monogenic diseases, genetic testing plays no practical role yet in the management of multifactorial cardiovascular diseases. Cell culture models based on induced pluripotent stem cells open the perspective of individualized testing of cardiovascular disease severity and pharmacological or genetic therapy. Biomarkers can identify individuals with an increased cardiovascular risk, and biomarker-guided therapy represents an attractive option, with troponin-guided therapy of acute coronary syndromes as a successful example [55]. Multiscale modeling of cardiac electrophysiology and mechanics for diagnosis as well as clinical decision support will facilitate the development of personalized cardiology, e.g., in arrhythmia and HF [56]. The introduction of new technologies such as high-resolution CT coronary imaging with fractional flow reserve, virtual reality, vascular robotic systems, and 3-D printing can be incorporated into personalized care to improve the efficacy as well as the safety of the treatment of patients with CVD [57]. Cardiologists can use their experience in personalized medicine to improve the efficiency of clinical research and provide more precise information to improve the health of individuals as well as populations [58]. In the future, artificial intelligence/ machine learning will likely assist physicians with differential diagnosis of the disease, suggest treatment options or recommendations, and provide cues for image interpretation to facilitate the practice of personalized medicine. Personalized approaches will gain increasing importance in the management of cardiovascular diseases in the future.
412

Med Princ Pract 2017;26:399-414 DOI: $10.1159 / 000481403$
Jain 


\section{References}

1 Jain KK: Textbook of Personalized Medicine, ed 2. New York, Springer, 2015.

2 Mirnezami R, Nicholson J, Darzi A: Preparing for precision medicine. N Engl J Med 2012; 366:489-491.

3 Jain KK: Applications of Biotechnology in Cardiovascular Therapeutics. New York, Springer, New York, 2011.

4 Yeh RW, Kramer DB: Decision tools to improve personalized care in cardiovascular disease: moving the art of medicine toward science. Circulation 2017;135:1097-1100.

5 Crosby J, Peloso GM, Auer PL, et al: Loss-offunction mutations in APOC3, triglycerides, and coronary disease. N Engl J Med 2014;371: $22-31$.

6 Jørgensen $A B$, Frikke-Schmidt R, Nordestgaard BG, Tybjærg-Hansen A: Loss-of-function mutations in APOC 3 and risk of ischemic vascular disease. N Engl J Med 2014;371: 32-41.

7 Graham MJ, Lee RG, Bell TA 3rd, et al: Antisense oligonucleotide inhibition of apolipoprotein C-III reduces plasma triglycerides in rodents, nonhuman primates, and humans. Circ Res 2013;112:1479-1490.

8 Nioi P, Sigurdsson A, Thorleifsson G, et al: Variant ASGR1 associated with a reduced risk of coronary artery disease. N Engl J Med 2016; 374:2131-2141.

9 Tybjærg-Hansen A: The sialylation pathway and coronary artery disease. N Engl J Med 2016;374:2169-2171.

10 Mega JL, Stitziel NO, Smith JG, et al: Genetic risk, coronary heart disease events, and the clinical benefit of statin therapy: an analysis of primary and secondary prevention trials. Lancet 2015;385:2264-2271.

11 Iakoubova $\mathrm{OA}$, Tong $\mathrm{CH}$, Rowland $\mathrm{CM}$, et al: Association of the Trp719Arg polymorphism in kinesin-like protein 6 with myocardial infarction and coronary heart disease in 2 prospective trials: the CARE and WOSCOPS trials. J Am Coll Cardiol 2008;51:435-443.

12 Shiffman D, Chasman DI, Zee RY, et al: A kinesin family member 6 variant is associated with coronary heart disease in the Women's Health Study. J Am Coll Cardiol 2008;51:444448.

13 Teekakirikul P, Kelly MA, Rehm HL, et al: Inherited cardiomyopathies: molecular genetics and clinical genetic testing in the postgenomic era. J Mol Diagn 2013;15:158-170.

14 D’Argenio V, Frisso G, Precone V, et al: DNA Sequence capture and next-generation sequencing for the molecular diagnosis of genetic cardiomyopathies. J Mol Diagn 2014;16: $32-44$.

15 Kathiresan S, Melander O, Anevski D, et al: Polymorphisms associated with cholesterol and risk of cardiovascular events. N Engl J Med 2008;358:1240-1249.
16 Tardif JC, Rhéaume E, Lemieux Perreault LP, et al: Pharmacogenomic determinants of the cardiovascular effects of dalcetrapib. Circ Cardiovasc Genet 2015;8:372-382.

17 Jain KK: Handbook of Biomarkers, ed 2. New York, Springer, 2017.

18 Jain KK: The role of nanobiotechnology in the development of personalized medicine. Med Princ Pract 2011;20:1-3.

19 Jain KK: Handbook of Nanomedicine, ed 3. New York, Springer, 2017.

20 Mangge H, Almer G, Stelzer I, et al: Laboratory medicine for molecular imaging of atherosclerosis. Clin Chim Acta 2014;437:19-24.

21 Saraste A, Nekolla SG, Schwaiger M: Cardiovascular molecular imaging: an overview. Cardiovasc Res 2009;83:643-652.

22 Chen HH, Josephson L, Sosnovik DE: Imaging of apoptosis in the heart with nanoparticle technology. Wiley Interdiscip Rev Nanomed Nanobiotechnol 2011;3:86-99.

23 Kumar A, Jena PK, Behera S, et al: Multifunctional magnetic nanoparticles for targeted delivery. Nanomedicine 2010;6:64-69.

24 Matsa E, Ahrens JH, Wu JC: Human induced pluripotent stem cells as a platform for personalized and precision cardiovascular medicine. Physiol Rev 2016;96:1093-1126.

25 Iqbal J, Serruys PW: Revascularization strategies for patients with stable coronary artery disease. J Intern Med 2014;276:336-351.

26 Carbone F, Montecucco F, Mach F: Update on evidence for treatment with ranolazine in stable angina. Swiss Med Wkly 2013;143: w13874.

27 O'Connor CM, Fiuzat M, Carson PE, et al: Combinatorial pharmacogenetic interactions of bucindolol and $\beta 1, \alpha 2 \mathrm{C}$ adrenergic receptor polymorphisms. PLoS One 2012; 7:e44324.

28 Maglo KN, Rubinstein J, Huang B, Ittenbach RF: BiDil in the clinic: an interdisciplinary investigation of physicians' prescription patterns of a race-based therapy. AJOB Empir Bioeth 2014;5:37-52.

29 Azizan EA, Poulsen H, Tuluc P, et al: Somatic mutations in ATP1A1 and CACNA1D underlie a common subtype of adrenal hypertension. Nat Genet 2013;45:1055-1060.

30 James PA, Oparil S, Carter BL, et al: $2014 \mathrm{ev-}$ idence-based guideline for the management of high blood pressure in adults: report from the panel members appointed to the Eighth Joint National Committee. JAMA 2014;311: 507-520.

31 SPRINT Research Group; Wright JT Jr, Williamson JD, et al: A randomized trial of intensive versus standard blood-pressure control. N Engl J Med 2015;373:2103-2116.

32 Sussman J, Vijan S, Hayward R: Using benefit-based tailored treatment to improve the use of antihypertensive medications. Circulation 2013;128:2309-2317.
33 Zhao FL, Zhang YZ, Tai GX, et al: Serum parathyroid hormone as a potential novel biomarker of coronary heart disease. Genet Test Mol Biomarkers 2014;18:670-674.

34 Floras JS: Blood pressure variability: a novel and important risk factor. Can J Cardiol 2013; 29:557-563.

35 Plachta DT, Gierthmuehlen M, Cota O, et al: Blood pressure control with selective vagal nerve stimulation and minimal side effects. J Neural Eng 2014;11:036011.

36 Vimaleswaran KS, Cavadino A, Berry DJ; LifeLines Cohort Study Investigators, et al: Association of vitamin D status with arterial blood pressure and hypertension risk: a mendelian randomisation study. Lancet Diabetes Endocrinol 2014;2:719-729.

37 Franceschini N, Fox E, Zhang Z, et al: Genome-wide association analysis of bloodpressure traits in African-ancestry individuals reveals common associated genes in African and non-African populations. Am J Hum Genet 2013;93:545-554.

38 Huan T, Esko T, Peters MJ, et al: A meta-analysis of gene expression signatures of blood pressure and hypertension. PLoS Genet 2015; 11:e1005035.

39 Hamlyn JM, Linde CI, Gao J, et al: Neuroendocrine humoral and vascular components in the pressor pathway for brain angiotensin II: a new axis in long term blood pressure control. PLoS One 2014;9:e108916.

40 Egan BM, Li J, Hutchison FN, Ferdinand KC Hypertension in the United States 19992012: progress Toward Healthy People 2020 goals. Circulation 2014;130:1692-1699.

41 Lonn EM, Bosch J, López-Jaramillo P, et al: Blood-pressure lowering in intermediate-risk persons without cardiovascular disease. N Engl J Med 2016;374:2009-2020.

42 Cushman WC, Goff DC: More HOPE for prevention with statins. N Engl J Med 2016;374: 2085-2087.

43 Liu X, Wang K, Wang L, Tsilimingras D: Microalbuminuria, macroalbuminuria and uncontrolled blood pressure among diagnosed hypertensive patients: the aspect of racial disparity in the NHANES study. Hypertens Res 2013;36:1100-1106.

44 Butt DA, Harvey PJ: Benefits and risks of antihypertensive medications in the elderly. J Intern Med 2015;278:599-626.

45 Ferrario CM, Jessup JA, Smith RD: Hemodynamic and hormonal patterns of untreated essential hypertension in men and women. Ther Adv Cardiovasc Dis 2013;7:293-305.

46 Fontana V, Luizon MR, Sandrim VC: An update on the pharmacogenetics of treating hypertension. J Hum Hypertens 2015;29:283291. 
47 Gerhard T, Gong Y, Beitelshees AL, et al: Alpha-adducin polymorphism associated with increased risk of adverse cardiovascular outcomes: results from GENEtic Substudy of the INternational VErapamil SR-trandolapril STudy (INVEST-GENES). Am Heart J 2008; 156:397-404.

48 Manunta P, Ferrandi M, Cusi D, et al: Personalized therapy of hypertension: the past and the future. Curr Hypertens Rep 2016;18:24.

49 Khera AV, Emdin CA, Drake I, et al: Genetic risk, adherence to a healthy lifestyle, and coronary disease. N Engl J Med 2016;375:23492358.

50 Chu P, Pandya A, Salomon JA, et al: Comparative effectiveness of personalized lifestyle management strategies for cardiovascular disease risk reduction. J Am Heart Assoc 2016;5:e02737.
51 Nes BM, Gutvik CR, Lavie CJ, et al: Personalized Activity Intelligence (PAI) for prevention of cardiovascular disease and promotion of physical activity. Am J Med 2017;130:328336.

52 Björnson E, Borén J, Mardinoglu A: Personalized cardiovascular disease prediction and treatment-a review of existing strategies and novel systems medicine tools. Front Physiol 2016;7:2.

53 Rolland T, Taşan M, Charloteaux B, et al: A proteome-scale map of the human interactome network. Cell 2014;159:1212-1226.

54 Blaus A, Madabushi R, Pacanowski M, et al: Personalized cardiovascular medicine today: a Food and Drug Administration/Center for Drug Evaluation and Research perspective. Circulation 2015;132:1425-1432.
55 Eschenhagen T, Blankenberg S: Personalized therapy in cardiology: biomarkers, pharmacogenetics and therapy of monogenic diseases. Internist (Berl) 2013;54:147-148, 150152, 154.

56 Zhang Y, Barocas VH, Berceli SA, et al: Multiscale modeling of the cardiovascular system: disease development, progression, and clinical intervention. Ann Biomed Eng 2016;44: 2642-2660.

57 Dugas CM, Schussler JM: Advanced technology in interventional cardiology: a roadmap for the future of precision coronary interventions. Trends Cardiovasc Med 2016;26:466473.

58 Antman EM, Loscalzo J: Precision medicine in cardiology. Nat Rev Cardiol 2016;13:591602. 